\title{
Power law Kohn anomalies and the excitonic transition in graphene
}

\author{
F. de Juan and H.A. Fertig \\ Department of Physics, Indiana University, Bloomington, IN 47405, USA
}

(Dated: September 10, 2018)

\begin{abstract}
Dirac electrons in graphene in the presence of Coulomb interactions of strength $\beta$ have been shown to display power law behavior with $\beta$ dependent exponents in certain correlation functions, which we call the mass susceptibilities of the system. In this work, we first discuss how this phenomenon is intimately related to the excitonic insulator transition, showing the explicit relation between the gap equation and response function approaches to this problem. We then provide a general computation of these mass susceptibilities in the ladder approximation, and present an analytical computation of the static exponent within a simplified kernel model, obtaining $\eta_{0}=\sqrt{1-\beta / \beta_{c}}$. Finally we emphasize that the behavior of these susceptibilities provides new experimental signatures of interactions, such as power law Kohn anomalies in the dispersion of several phonons, which could potentially be used as a measurement of $\beta$.
\end{abstract}

\section{INTRODUCTION}

One of the most intriguing aspects of graphene ${ }^{1}$ continues to be the physics of the electron-electron interaction and its effects on the electronic properties. This very active field of research ${ }^{2}$ is now of great relevance as current experiments are displaying unambiguous signatures of interactions ${ }^{3,4}$.

What makes this problem so interesting, among other reasons, is the fact that graphene, when undoped, is a rather special material with respect to the Coulomb interaction. Because the low-energy quasiparticles of this system are massless Dirac fermions, with vanishing density of states at the Fermi level, the $1 / r$ Coulomb interaction remains long ranged even in the presence of screening. This makes this system very different from materials with a finite Fermi surface, and gives rise to very unusual many-body effects. This behavior stems from the fact that the Hamiltonian of Coulomb interacting Dirac fermions does not contain an intrinsic energy scale. The kinetic and interaction terms scale in the same way, and the Hamiltonian is in fact scale invariant. In the RG sense, the Coulomb interaction is marginal in this system ${ }^{5}$. The strength of the Coulomb interaction, the fine structure constant of graphene, may be expressed as a dimensionless number $\beta=e^{2} /\left(\epsilon v_{F}\right)$, where $v_{F}$ is the Fermi velocity of the Dirac quasiparticles, $e$ their charge, and $\epsilon$ the dielectric constant of the substrate. While a naive estimate gives an upper limit $\beta \approx 2$ for $\epsilon=1$, implying that Coulomb interactions should be relatively important, experimentally their strength is still a matter of debate ${ }^{3,4}$.

The presence of this interaction has many non-trivial consequences. From a weak coupling perspective, the first to be predicted was the logarithmic renormalization of the Fermi velocity ${ }^{5}$, which has been recently measured ${ }^{4}$. Other observables have been shown to be affected by a similar renormalization ${ }^{2,6}$. An electronic inverse lifetime that is linear instead of quadratic with energy $^{7}$ is also a characteristic feature of this system.

Another interesting phenomenon induced by interactions is the possibility of a phase transition to a broken symmetry state in the strong coupling regime. For a strong enough value of the coupling $\beta$, low energy electrons and holes may bind into excitons, opening a gap in the system. The broken symmetry state is thus known as the excitonic insulator, and the characteristics of this transition have been studied throughly ${ }^{8-16}$. The critical coupling is thought to be of order $\beta \approx 1$ but its precise value is still under discussion $11-16$, and experimentally an intrinsic gap in graphene has not been observed ${ }^{4}$. This excitonic transition can also be considered as the manybody counterpart of the supercritical screening of an external Coulomb potential by Dirac fermions ${ }^{17,18}$.

A more recent prediction of an interaction induced phenomenon, also rooted in the scale invariance of this system, is the presence of power law behavior with interaction dependent exponents in certain correlation functions ${ }^{12,18-21}$, as if the system were in a critical phase. These correlators can be considered the mass-mass response functions (or mass susceptibilities) of the system. In analogy to the charge-charge response function, which measures the charge expectation value as a response to an external perturbation coupling to the charge, this susceptibility is the analog quantity built with mass vertices (i.e. the matrices that gap the Dirac spectrum) instead of charge ones. The experimental detection of these correlations would represent a new signature of interaction effects beyond those already discussed, and would be highly desirable. In addition, the singularities of the mass susceptibilities are directly related to instabilities to spontaneous mass generation, and therefore their observation will shed light on the problem of the excitonic transition. This observation is however challenging, as there is no simple experiment to directly probe a mass (as opposed to charge or spin) susceptibility.

The objective of this work is twofold. First we will show the explicit correspondence between the physics of the excitonic transition and the behavior of the mass susceptibilities. We will illustrate it in detail by comparing the transition as seen from a gap equation and a response function perspective. Second, we will argue that there are in fact current experiments available to observe the mass susceptibilities. We will see that there are clear 
experimental signatures of their presence in the dispersion relation of certain phonons, which acquire power law Kohn anomalies with $\beta$ dependent exponents. In addition, we will show that the static exponent of the mass susceptibility, obtained within perturbative $\mathrm{RG}^{12,20,21}$, or numerically $\stackrel{18,19}{1}$, can in fact can be obtained analytically from the ladder approximation and is given by $\sqrt{1-\beta / \beta_{c}}$.

The paper is organized as follows. In Sec. Iwe review the physics of the excitonic transition and its description in terms of a gap equation. In Sec. III we describe the general mass-mass response function and compute it in different approximations. In Sec. IV] we discuss how the mass susceptibility may be observed in the dispersion relation of particular phonons, and in Sec. $\mathrm{V}]$ we present our conclusions.

\section{THE EXCITONIC TRANSITION}

\section{A. The model}

We start by reviewing the excitonic insulator transition in graphene. We will consider spinless graphene for simplicity, as spin will not play any role and can be accounted for when necessary. In graphene, the low energy excitations around the $K$ and $K^{\prime}$ points can be modeled in terms of a Dirac Hamiltonian in two spatial dimensions

$$
H=i v_{F} \int d^{2} r \psi^{\dagger}\left(\alpha_{x} \partial_{x}+\alpha_{y} \partial_{y}\right) \psi,
$$

with $\vec{\alpha}=\left(\tau_{z} \sigma_{x}, \sigma_{y}\right)$, where the $\sigma$ and $\tau$ matrices act on the sublattice and valley degrees of freedom, respectively. The Coulomb interaction is included as

$$
H_{i n t}=\frac{e^{2}}{2} \int d^{2} r d^{2} r^{\prime} \frac{\psi_{r}^{\dagger} \psi_{r} \psi_{r^{\prime}}^{\dagger} \psi_{r^{\prime}}}{\left|\vec{r}-\vec{r}^{\prime}\right|}
$$

This Hamiltonian has an $\mathrm{SU}(2)$ valley symmetry generated by the matrices $T_{n}=\left(\tau_{x} \sigma_{y}, \tau_{y} \sigma_{y}, \tau_{z}\right)$, in the sense that the $\mathrm{SU}(2)$ rotation $\psi \rightarrow e^{i T_{n} \theta_{n}} \psi$ leaves the Hamiltonian invariant 22 .

When $\beta$ is large enough, this system has an instability towards the pairing of electrons and holes. This pairing generates a mass for the Dirac fermions, opening a gap in the spectrum, and so this state is known as the excitonic insulator. This instability is signaled by the development of a finite expectation value of a mass operator $\left\langle\psi^{\dagger} M \psi\right\rangle$. There are four possible mass matrices $M$ for the Hamiltonian (1), which correspond to different microscopic mechanisms for the instability, and which break different symmetries. The first of them is the generation of a charge imbalance between sublattices (a charge density wave), and corresponds to a matrix $\sigma_{z}$. The next two are produced by a bond density wave, the Kekulé distortion ${ }^{23}$, and correspond to the matrices $\tau_{x} \sigma_{x}$ and $\tau_{y} \sigma_{x}$. Finally, a pattern of circulating currents in the unit cell gives rise to the mass $\sigma_{z} \tau_{z}$, known as the Haldane mass.
The Kekulé and CDW masses are time reversal invariant, and may be grouped into the three components of a spin $1 / 2$ vector

$$
M_{n}=\left(\tau_{x} \sigma_{x}, \tau_{y} \sigma_{x}, \sigma_{z}\right),
$$

because they transform as such under the $\mathrm{SU}(2)$ valley symmetry. Therefore, when one of them is generated, the valley symmetry is spontaneously broken to $\mathrm{U}(1)$, analogously to a ferromagnet. The Haldane mass, on the other hand, is a scalar under valley symmetry but breaks time reversal. In this work we will be concerned only with the time reversal invariant masses and valley symmetry breaking. The structure of these masses and the symmetries they break is analogous to the one found in QED3, where the valley symmetry is known as chiral symmetry. In fact, the problem of chiral symmetry breaking and mass generation in QED3 in the $1 / N$ approximation 24.25 has much in common with the excitonic transition, the main difference being that the interaction in QED3 is a Lorentz invariant gauge field (rather than an instantaneous charge-charge interaction).

\section{B. The gap equation}

The most common approach to show the existence of the excitonic transition is by means of a self-consistent gap equation $\underline{\underline{8}-14}$. This is equivalent to the Hartree-Fock or mean field approximation. We will now review the main features of this approach, with the aim of highlighting its relation with the response function approach to be discussed in the next section. For simplicity we set $v_{F}=1$ henceforth and thus the Coulomb coupling $e^{2}=\beta$.

To derive the gap equation, the full electron propagator $G$ is expressed in terms of the self-energy as $G^{-1}=G_{0}^{-1}-$ $\Sigma$, with the bare propagator

$$
G_{0}(k)=\frac{k_{0}+\vec{\alpha} \vec{k}}{k_{0}^{2}-k^{2}+i \epsilon} .
$$

The self-energy is a $4 \mathrm{x} 4$ matrix that satisfies the Schwinger-Dyson equation

$$
\Sigma(p)=-i \int \frac{d^{3} k}{(2 \pi)^{3}} \tilde{\Gamma}^{0}(k, p) \tilde{\Pi}(p-k)\left(G_{0}^{-1}-\Sigma\right)^{-1},
$$

where $G, \tilde{\Pi}$ are the full electron and photon propagators, and $\tilde{\Gamma}^{0}$ the full Coulomb vertex. This equation is so far exact, but to solve it one needs to assume approximate forms for $\tilde{\Pi}$ and $\tilde{\Gamma}^{0}$. The simplest approximation is to assume a bare Coulomb vertex $\tilde{\Gamma}^{0}=1$ and a bare photon $\tilde{\Pi}=2 \pi \beta /|\vec{q}|$, which gives

$$
\Sigma(p)=-i \beta \int \frac{d^{3} k}{(2 \pi)^{2}} \frac{1}{|\vec{p}-\vec{k}|}\left(G_{0}^{-1}-\Sigma\right)^{-1} .
$$

This equation can be seen as the resummation of the "rainbow" diagrams, depicted diagrammatically in Fig. 
1. To solve this equation $\Sigma$ can be expanded in a basis of all sublattice and valley matrices. We will make the further approximation that only terms proportional to mass matrices are important (neglecting Fermi velocity and wavefunction renormalizations) i.e. we assume that

$$
\Sigma_{i j}=\Delta_{n}\left(M_{n}\right)_{i j},
$$

where summation over repeated indices is always implicit. Projecting into each mass channel (i.e. taking the trace of Eq. (6) with $M_{m}$ ) we arrive at

$$
\Delta_{m}(p)=-i \beta \delta_{m n} \int \frac{d^{3} k}{(2 \pi)^{2}} \frac{\Delta_{n}(k)}{|\vec{p}-\vec{k}|} \frac{1}{k_{0}^{2}-k^{2}-\Delta_{n}(k)^{2}} .
$$

While the gap equations for the three masses can be studied separately, we see that they are in fact related by symmetry, so that we can drop the index $n$. Because the interaction is $k_{0}$ independent in this approximation, the gap is too, and we may integrate over $k_{0}$ to obtain

$$
\Delta(p)=\pi \beta \int \frac{d^{2} k}{(2 \pi)^{2}} \frac{\Delta(k)}{|\vec{p}-\vec{k}|} \frac{1}{\sqrt{k^{2}+\Delta(k)^{2}}} .
$$

This is the simplest version of the gap equation for the excitonic problem. More refined approximations have been considered in the literature, for example at finite temperature ${ }^{8}$, with the renormalization of the fermion spectrum $10,11,13$, or with statio $\underline{14,17}$ and dynamio 12 RPA $(1 / \mathrm{N})$ screening for the interaction. In general, all approaches agree that there is an excitonic transition, but critical couplings vary significantly. For our discussion we retain the simplest form given by Eq. (9).

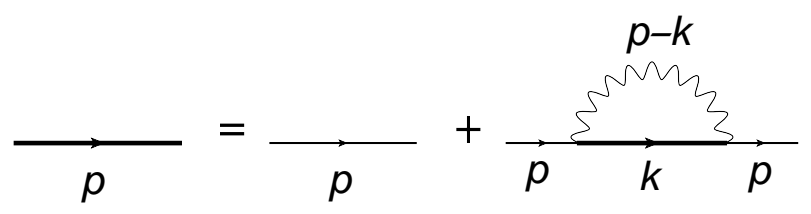

FIG. 1. Self consistent diagrammatic equation for the electron Green function (full line) in the rainbow approximation

To make further progress, the standard approach is to implement an expansion of the gap in circular harmonics,

$$
\Delta^{\left(n_{p}\right)}=\int \frac{d \theta_{p}}{2 \pi} e^{i n_{p} \theta_{p}} \Delta(p)
$$

and keeping only the first order term, $\Delta=\Delta^{(0)}$, which is equivalent to assuming that $\Delta$ has no angle dependence. The equation is now expressed as

$$
\Delta(p)=\frac{\beta}{2 p} \int k d k \frac{\Delta(k)}{\sqrt{k^{2}+\Delta(k)^{2}}} C^{(0)}(k / p),
$$

where the Coulomb kernel is defined as

$$
C^{(n)}(x)=\int \frac{d \theta_{k}}{2 \pi} \frac{e^{i n \theta_{k}}}{\left(1+x^{2}+2 x \cos \theta_{k}\right)^{1 / 2}},
$$

This integral equation can be solved numerically by iteration, but it is instructive to discuss first an analytical solution that is available when a simplified version of the kernel is taken, given by

$$
C^{(0)}(x)=\theta(1-x)+\frac{1}{x} \theta(x-1),
$$

which has the correct asymptotic behavior at large and small arguments. The Coulomb kernel and its approximate form, as well as higher order kernels that are neglected, are plotted in Fig. 2 for comparison. The ad-

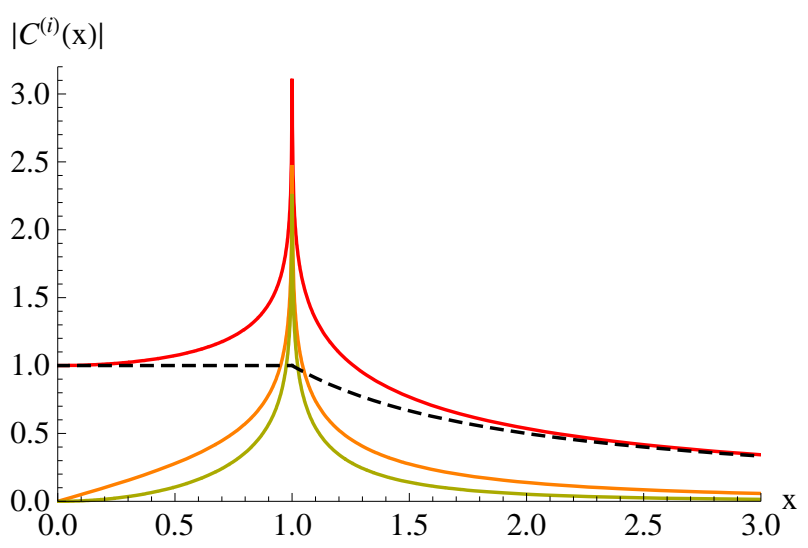

FIG. 2. Plots of the first three circular harmonics of the Coulomb kernel $C^{(0)}(k / p)$, Eq. (12). The corresponding simplified kernel given in Eq. (13) is shown for comparison (dashed line).

vantage of this approximate kernel is that it allows one to re-express the integral equation as a differential one. Taking two derivatives of Eq. (11) with respect to $p$, we obtain

$$
p^{2} \frac{\partial^{2} \Delta}{\partial p^{2}}+2 p \frac{\partial \Delta}{\partial p}+\frac{\beta}{2} \frac{p \Delta}{\sqrt{p^{2}+\Delta^{2}}}=0 .
$$

The boundary conditions for this equation are obtained from the first derivatives of Eq. (11) at the endpoints of the integration region

$$
\begin{array}{r}
p \frac{\partial \Delta}{\partial p}+\left.\Delta\right|_{p=\Lambda}=0 \\
\left.p^{2} \frac{\partial \Delta}{\partial p}\right|_{p=\mu}=0,
\end{array}
$$

where $\mu$ is an infrared cutoff (the inverse system size). Eq. (14) cannot be solved analytically, but one may linearize it to obtain the branching points in $\beta$ where nontrivial solutions become possible $\stackrel{8,12}{ }$,

$$
p^{2} \frac{\partial^{2} \Delta}{\partial p^{2}}+2 p \frac{\partial \Delta}{\partial p}+\frac{\beta}{2} \Delta=0 .
$$

This equation is the well known Schroedinger equation with a $1 / r^{2}$ potential ${ }^{26}$. Its solution is given by

$$
\Delta=A_{+} \tilde{p}^{\eta_{+}}+A_{-} \tilde{p}^{\eta_{-}},
$$


with $\eta_{ \pm}=1 / 2(1 \pm \gamma), \gamma=\sqrt{1-2 \beta}$ and all quantities with a tilde are scaled with the cutoff $\tilde{p}=p / \Lambda$. Applying the infrared condition (Eq. 16) we get

$$
\Delta=A\left[\eta_{-} \tilde{p}^{\eta_{+}}-\eta_{+} \tilde{\mu}^{\gamma} \tilde{p}^{\eta_{-}}\right] .
$$

The ultraviolet condition (Eq. 15) is also homogeneous and so does not fix the scale of the gap $A$, something that can only be done with the non-linear equation. The ultraviolet condition gives is the set of branching points where non-vanishing solutions of the gap equation become possible

$$
\sqrt{2 \beta-1} \log \tilde{\mu}=2 \arctan \left(\frac{\sqrt{2 \beta-1}}{\beta-1}\right)+2 n \pi,
$$

which represents an infinite number of logarithmically spaced solutions $\beta_{c}^{(i)}$ for $\beta>1 / 2$. (When $\mu \rightarrow 0$ all solutions collapse to $\beta_{c}=1 / 2$, although there is still one of lowest energy $\left.{ }^{9}\right)$. Note however that for $\beta$ greater than $\beta_{c}^{(0)}$, a finite gap is always present and the linearized equation cannot be used. The rest of the solutions thus represent higher order instabilities that would take place if the first one is set artificially to zero. Coming back to the full integral equation, Eq. (11), we can solve it by iteration procedures, and obtain both the gap and its dependence on $\beta$, which are plotted in Fig. 3. We observe that, for $\tilde{\mu}=10^{-10}$, a finite gap is generated for $\beta>\beta_{c}^{(0)} \approx 0.48$, which is the signature of the excitonic transition. From now on we will simply call $\beta_{c} \equiv \beta_{c}^{(0)}$, the physically relevant critical coupling.

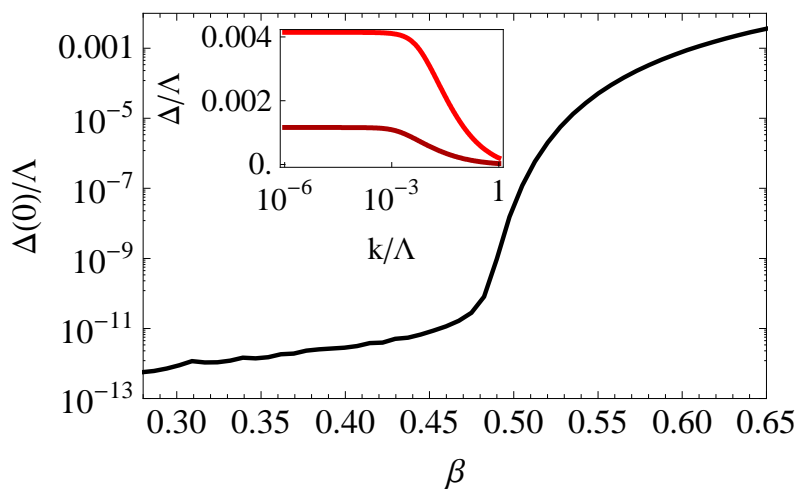

FIG. 3. Dependence of the gap $\Delta(0)$ on $\beta$ for $\tilde{\mu}=10^{-10}$. The gap becomes significant within numerical precision for $\beta \gtrsim 0.48$. Note that this computation is done with the full kernel $C^{(0)}$ and $\beta_{c}^{(0)}$ need not be greater than $1 / 2$. Inset: Momentum dependence of the gap $\Delta(p)$, for $\beta=0.61$ (lower curve) and $\beta=0.65$ (upper curve), computed with Eq. (11).

\section{RESPONSE FUNCTIONS}

A complementary approach to study the excitonic transition is through response functions. These are defined as the expectation value of a mass operator $\psi^{\dagger} M \psi$ expanded to first order in an external mass perturbation. They can be considered as generalized susceptibilities in the mass channel, and their singularities represent the instabilities of the system to spontaneous mass generation. Therefore they represent an alternative approach to study the excitonic transition. However, the importance of the response functions goes beyond the characterization of the transition, as they represent physical observables with non-trivial interaction signatures also for $\beta<\beta_{c}$. This has also been emphasized in the context of the $\mathrm{QED}_{3}$ theory of the cuprates ${ }^{27-30}$, where the behavior of the mass susceptibilities is similar to what is found in graphene.

The response in the $M_{n}$ channel to a perturbation $M_{m}$ is simply the correlator

$$
\Pi_{n m}(q)=2 i \int \frac{d^{3} p}{(2 \pi)^{3}} \operatorname{tr}\left[M_{n} G(p) \Gamma_{m}(p, p+q) G(p+q)\right],
$$

which is shown diagrammatically in Fig. 4(b), and where $\Gamma_{m}$ is the full mass vertex (note that $\Gamma_{m}$ is a $4 \times 4$ matrix; the sublattice/valley index is omitted for clarity) and the factor of 2 accounts for spin. Again, this equation is exact but to solve it we need to approximate $\Gamma$ in some way. To guide us in the choice of approximation, we realize that the full mass vertex satisfies the following property: if we include an external mass $m_{n} \psi^{\dagger} M_{n} \psi$ in the Hamiltonian, then by construction it holds that

$$
\left.\frac{\partial G(p)}{\partial m_{n}}\right|_{m_{n}=0}=\left.G(p) \Gamma_{n}(p, p) G(p)\right|_{m_{n}=0},
$$

or equivalently in terms of the self-energy and writing the indices explicitly

$$
\left(M_{n}\right)_{i j}+\left.\frac{\partial \Sigma_{i j}(p)}{\partial m_{n}}\right|_{m_{n}=0}=\left.\left(\Gamma_{n}\right)_{i j}(p, p)\right|_{m_{n}=0} .
$$

This identity can be proven diagrammatically by realizing the derivative acts by cutting all possible fermion lines in the full self-energy and introducing a mass vertex $M_{n}$ at every cut. If we assumed the rainbow summation for the self-energy, it can be seen that the corresponding approximation for $\Gamma$ is the ladder approximation, depicted in Fig. 4(a). In this case $\Gamma$ satisfies the self-consistent equation

$$
\Gamma_{m}(p, q)=M_{m}+i \beta \int \frac{d^{3} k}{(2 \pi)^{2}} \frac{G(k) \Gamma_{m}(k, q) G(k+q)}{|\vec{p}-\vec{k}|} .
$$

It is important to note that the propagators in this equation are the full propagators in the rainbow approximation. These are just $G_{0}$ for $\beta<\beta_{c}$ as shown in the previous section, but acquire a mass for $\beta>\beta_{c}$ and this has to be included to have a consistent computation. This simply represents the fact that after the phase transition, the propagators have to be computed in the broken symmetry state. Note also that, as in what happens in 
the gap equation, we anticipate by symmetry that the response function will satisfy $\Pi_{n m}=\delta_{n m} \Pi$, so we can drop the indices in this case too.

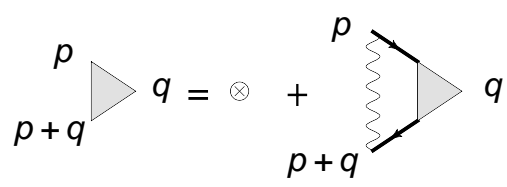

(a)

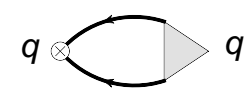

(b)
FIG. 4. (a) Diagrammatic equation for the three point vertex (shaded triangle) in the ladder approximation. The cross denotes a mass vertex. (b) Response function diagram.

We will now compute the response and vertex functions, and for the sake of clarity we will do so in two steps. To have some insight into the excitonic transition itself, it is simplest to discuss just $\Pi\left(q_{0}=0, q=0\right)$. In addition the computation for $\beta>\beta_{c}$ presents no major difficulty. Our main interest, however, is the full response function $\Pi(\omega, q)$, which will compute next in what appears to be the experimentally relevant case of $\beta<\beta_{c}$. The extension of $\Pi(\omega, q)$ to $\beta>\beta_{c}$ is also feasible but it is beyond the scope of this work.

\section{A. $\Pi(0,0)$ and the excitonic transition}

The computation of $\Pi(0,0)$ is simplified by the identity in Eq. (23). Since in the previous section we assumed $\Sigma_{i j}=\Sigma_{n}\left(M_{n}\right)_{i j}$, Eq. (23) implies that that $\Gamma_{n}(p, p)$ is also proportional to the corresponding mass matrix, $\left(\Gamma_{n}\right)_{i j}=\left(M_{n}\right)_{i j} \Gamma$. This allows us to perform the traces easily in both Eqs. (21) and (24). Plugging in $G$ as obtained in the previous section, and integrating in energy, the equations read

$$
\begin{gathered}
\Pi(q)=4 \int \frac{d^{2} p}{(2 \pi)^{2}} \frac{p^{2}}{\left(p^{2}+\Delta^{2}\right)^{3 / 2}} \Gamma(p, p), \\
\Gamma(p, p)=1+\pi \beta \int \frac{d^{2} k}{(2 \pi)^{2}} \frac{k^{2}}{\left(k^{2}+\Delta^{2}\right)^{3 / 2}} \frac{1}{|\vec{k}-\vec{p}|} \Gamma(k, k) .
\end{gathered}
$$

As for the gap equation, we now assume that $\Gamma$ has no angle dependence (i.e. we take only the first order of the expansion in circular harmonics) we find

$$
\begin{aligned}
\Pi(q) & =\frac{2}{\pi} \int d p \frac{p^{3}}{\left(p^{2}+\Delta^{2}\right)^{3 / 2}} \Gamma(p, p) \\
\Gamma(p, p) & =1+\frac{\beta}{2 p} \int d k \frac{k^{3}}{\left(k^{2}+\Delta^{2}\right)^{3 / 2}} C^{(0)}(k / p) \Gamma(k, k) .
\end{aligned}
$$

A direct computation shows that Eq. (28) can also be obtained by adding an external mass $m_{n}$ to the propagator of Eq. (6) and taking the derivative with respect to it, as Eq. (23) mandates.
We are now ready to make the equivalence between the gap equation and the response function approaches explicit. Consider Eq. (28) for $\Delta=0$. If we write the equation for $\Gamma$ in the form

$$
\int d k A(k, p) \Gamma(k, k)=1,
$$

then

$$
A(k, p)=\delta(k-p)-\frac{\beta}{2 p} C^{(0)}(k / p) .
$$

We can obtain $\Gamma$ by inverting the operator $A(k, p)$, i.e. $\Gamma=A^{-1}$. On the other hand, the linearized version of the gap equation has the form

$$
\int d k A(k, p) \Delta(k)=0,
$$

in terms of the same operator. Therefore, whenever there is a non-vanishing solution of the linearized gap equation $A(k, p)$ develops a zero eigenvalue, its inverse becomes singular, and $\Gamma$ develops a divergence. Therefore the response function $\Pi(0,0)$, which is the just the integral of $\Gamma$, also develops divergences at the $\beta_{c}^{(i)}$ whenever the linearized gap equation has a solution, i.e. at the critical points for the different instabilities.

The computation of $\Pi(0,0)$ with $\Delta$ set to zero was carried out in Ref. 18, where it was proven that an infinite number of logarithmically spaced poles appear for $\beta>\beta_{c}$. This result is reproduced in Fig. 5. Moreover, the analytical solution in terms of the model kernel showed that these poles are in fact given also by our Eq. (20) for the gap equation, as they should. The artificial constraint $\Delta=0$ may therefore be used to locate the critical couplings for higher order solutions of the gap equation. Physically, however, as long as we cross $\beta_{c}$ and the gap is generated, the system always stays in the lowest energy ground state and no further poles should be observed. This is indeed what is obtained from the numerical solution of Eq. (28) if $\Delta$ is included as computed from Eq. (11). In Fig. 5 we show both cases for comparison.

\section{B. General computation of $\Gamma$ and $\Pi$}

We now proceed to solve the general equations (21) and (24). We will only consider $\beta<\beta_{c}$ for simplicity, i.e., we set $\Delta=0$ for the rest of the article. To solve this set of equations, it is convenient to decompose $\Gamma_{m}$ in a basis of $4 \times 4$ matrices with well defined transformation properties under the $\mathrm{SU}(2)$ valley symmetry. Defining $\tilde{M}=\tau_{z} \sigma_{z}$, this basis may be taken as the four matrices $\tilde{M}, \mathcal{I}, \alpha^{i}$ which are scalars under this symmetry, and the matrices $M_{n}, T_{n}, \alpha^{i} T_{n}$, each of which transforms like a spin $1 / 2$. With this choice we express $\Gamma_{m}$ as

$$
\begin{aligned}
\Gamma_{m} & =\tilde{\Gamma}_{m} \tilde{M}+\tilde{\Gamma}_{m}^{0} \mathcal{I}+\tilde{\Gamma}_{m}^{i} \alpha^{i} \\
& +\Gamma_{n m} M_{n}+\Gamma_{n m}^{0} T_{n}+\Gamma_{n m}^{i} \alpha^{i} T_{n}
\end{aligned}
$$




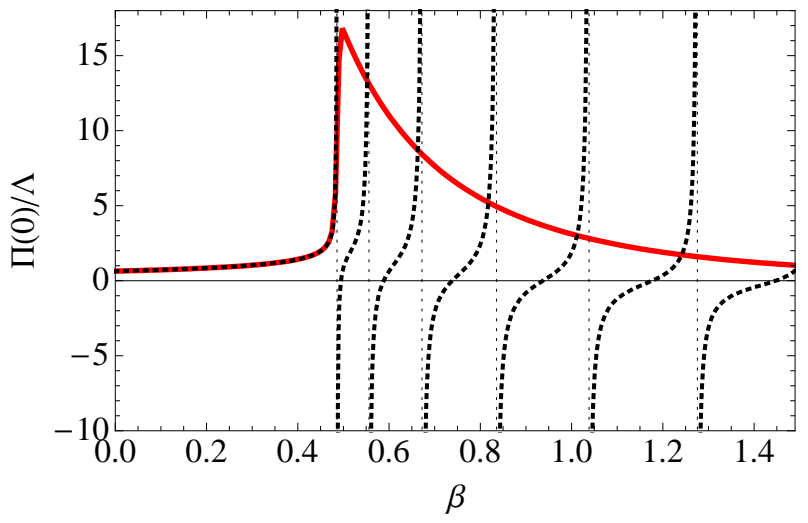

FIG. 5. Zero momentum response function $\Pi(0,0)$ as a function of $\beta$. When the gap is not included in the electron propagators, the result presents logarithmically spaced poles (dotted black line). Asymptotes are shown (thin dotted lines) where poles occur. When self-energy diagrams are included in the fermion propagators, the generation of a mass prevents the appearance of poles after the transition (full red line). Note the first pole is approximately located at the critical coupling obtained from the gap equation.

The equations are further simplified when $\Gamma_{n m}^{i}$ is expressed in terms of its longitudinal and transverse parts

$$
\Gamma_{n m}^{L}=\hat{q} \cdot \vec{\Gamma}_{n m}, \quad \Gamma_{n m}^{T}=\hat{q} \times \vec{\Gamma}_{n m},
$$

where $\hat{q}=\vec{q} / q$. A similar relation applies for $\tilde{\Gamma}_{n m}^{i}$. With the identities

$$
\begin{aligned}
\vec{k} \cdot \vec{\Gamma}_{n m} & =\vec{k} \cdot \hat{q} \Gamma_{n m}^{L}-\vec{k} \times \hat{q} \Gamma_{n m}^{T}, \\
\vec{k} \times \vec{\Gamma}_{n m} & =\vec{k} \cdot \hat{q} \Gamma_{n m}^{T}+\vec{k} \times \hat{q} \Gamma_{n m}^{L},
\end{aligned}
$$

substituting Eq. (32) into Eq. (21), and performing the trace, we obtain

$$
\begin{aligned}
\Pi_{n m}(q)=i \int & \frac{d^{3} p}{(2 \pi)^{3}} \frac{8}{D}\left[f_{11} \Gamma_{n m}+f_{12} \Gamma_{n m}^{T}\right. \\
& \left.+\vec{p} \times \vec{q}\left(f_{13} \Gamma_{n m}^{L}+f_{14} \Gamma_{n m}^{0}\right)\right]
\end{aligned}
$$

where we have defined the denominator

$$
D(p, q)=\left[p_{0}^{2}-\vec{p}^{2}+i \epsilon\right]\left[\left(p_{0}+q_{0}\right)^{2}-(\vec{p}+\vec{q})^{2}+i \epsilon\right],
$$

and where all $f_{i j}(\vec{p}, \vec{q})$ (specified below) are even functions under the reversal of the relative angle $\theta_{\vec{p}, \vec{q}}=$ $\theta_{p}-\theta_{q}$. Because of the decomposition in Eq. (32), the scalar parts decouple completely and are not needed. We can then obtain equations for the relevant components of $\Gamma_{m}$ by multiplying Eq. (24) by the corresponding basis matrices and taking the trace. One then obtains

$$
\begin{aligned}
\Gamma_{n m} & =\delta_{n m}-i \beta \int \frac{d^{3} k}{(2 \pi)^{2}} \frac{1}{D} \frac{1}{|\vec{p}-\vec{k}|}\left[f_{11} \Gamma_{n m}+f_{12} \Gamma_{n m}^{T}\right. \\
& \left.+\vec{k} \times \vec{q}\left(f_{13} \Gamma_{n m}^{L}-f_{14} \Gamma_{n m}^{0}\right)\right] \\
\Gamma_{n m}^{T} & =-i \beta \int \frac{d^{3} k}{(2 \pi)^{2}} \frac{1}{D} \frac{1}{|\vec{p}-\vec{k}|}\left[f_{21} \Gamma_{n m}+f_{22} \Gamma_{n m}^{T}\right. \\
& \left.\left.+\vec{k} \times \vec{q}\left(f_{23} \Gamma_{n m}^{L}+f_{24} \Gamma_{n m}^{0}\right)\right)\right] .
\end{aligned}
$$

$\Gamma_{m n}^{L}$ and $\Gamma_{m n}^{0}$ satisfy similar equations, but are not needed in what follows. We now perform a circular harmonic expansion

$$
\Gamma^{\left(n_{p}, n_{q}\right)}=\int \frac{d \theta_{p}}{2 \pi} e^{i n_{p} \theta_{p}} \frac{d \theta_{q}}{2 \pi} e^{i n_{q} \theta_{q}} \Gamma(p, q),
$$

and retain only the first order contribution. Terms containing $\vec{k} \times \vec{q}$ are odd and vanish. Thus, $\Gamma_{n}^{L}$ and $\Gamma_{n}^{0}$ completely decouple to first order. As anticipated, from the structure of Eqs. (36), (38) and (39) it can be seen that in fact $\Pi_{n m}=\delta_{n m} \Pi$. With this simplification the relevant components of $f_{i j}$ are

$$
\begin{aligned}
& f_{11}=-k_{0}\left(k_{0}+q_{0}\right)+\vec{k}(\vec{k}+\vec{q}), \\
& f_{12}=f_{21}=i\left(\frac{q_{0} \vec{k} \vec{q}}{q}-k_{0} q\right), \\
& f_{22}=\frac{2(\vec{q} \times \vec{k})^{2}}{q^{2}}+k_{0}\left(k_{0}+q_{0}\right)-\vec{k}(\vec{k}+\vec{q}) .
\end{aligned}
$$

Defining

$$
K_{i j}^{(n)}=\frac{i}{\pi} \int \frac{d \theta_{p}}{2 \pi} e^{\theta_{p} n} \int d k_{0} k \frac{f_{i j}}{D},
$$

the self-consistent equations to first order in the circular harmonic expansion finally read

$$
\begin{gathered}
\Gamma^{(0,0)}=1+\frac{\beta}{2 p} \int d k C^{(0)}\left(K_{11}^{(0)} \Gamma^{(0,0)}+K_{12}^{(0)} \Gamma_{T}^{(0,0)}\right), \\
\Gamma_{T}^{(0,0)}=-\frac{\beta}{2 p} \int d k C^{(0)}\left(K_{21}^{(0)} \Gamma^{(0,0)}+K_{22}^{(0)} \Gamma_{T}^{(0,0)}\right),
\end{gathered}
$$

where the Coulomb kernel $C^{(n)}$ was defined in Eq. (12). The mixing Kernel $K_{12}$, as well as the higher order harmonics of the kernel $K_{11}$ can be shown to be small and may be neglected. In this case, the final equations determining the response function, spelling momenta explicitly, are

$$
\begin{aligned}
\Gamma^{(0,0)}(p, q) & =1+\frac{\beta}{2 p} \int d k C^{(0)}(k / p) K_{11}^{(0)}(k, q) \Gamma^{(0,0)}(k, q) \\
\Pi(q) & =\frac{2}{\pi} \int d p K_{11}^{(0)}(p, q) \Gamma^{(0,0)}(p, q) .
\end{aligned}
$$


When the external $q<q_{0}$, all $K_{i j}$ develop an imaginary part for $\left(q_{0}-q\right) / 2<k<\left(q_{0}+q\right) / 2$. Note that when $\omega=q=0, K_{11}^{(0)}(k, q)=1$ and we recover Eq. (28) for $\Delta=0$.

\section{Analytic solution for the static vertex and response function for $\beta<\beta_{c}$}

In this section, we show how equations (47) and (48) can be solved analytically in the static limit $q_{0}=0$, if one assumes simplified versions for the kernels in the spirit of the previous section. This type of solution is also related to the one employed in the Lorentz invariant case in QED3 in the computation of the propagator of fermionantifermion composites 27 (the analog of excitons in our case). The inclusion of an infrared cutoff in this approximation makes it excessively complicated, so we will set $\mu=0$ for this section. is

The explicit expression for the static kernel in Eq. (47)

$$
K_{11}^{(n)}(k / q)=\int \frac{d \theta_{k}}{2 \pi} \frac{e^{i n \theta_{k}} k}{\vec{q}^{2}+2 \vec{q} \vec{k}}\left(\frac{\vec{k} \vec{q}}{k}+\frac{\vec{q}(\vec{k}+\vec{q})}{|\vec{k}+\vec{q}|}\right) .
$$

Note we can write $K_{11}^{(0)}(k, q)=K_{11}^{(0)}(k / q)$ when $q_{0}=0$. We will use the following simplified version

$$
K_{11}^{(0)}(x)=x \theta(1-x)+\theta(x-1),
$$

which is compared with the actual kernel and its higher order harmonics that are neglected in Fig. 6.

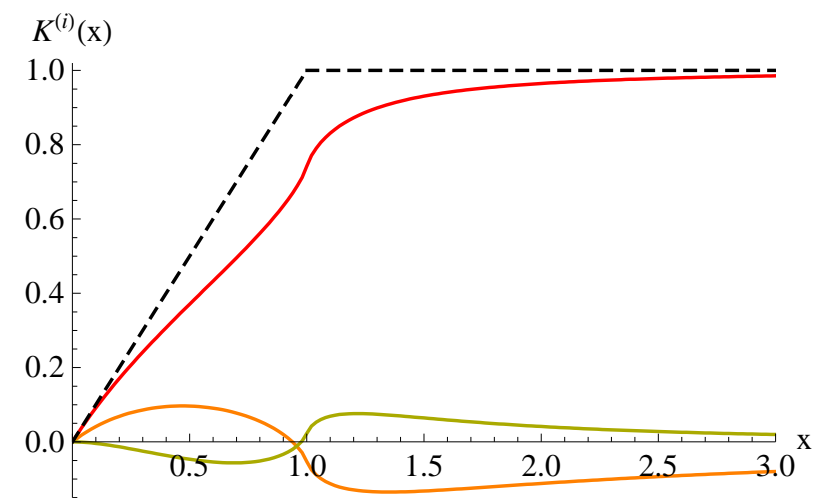

FIG. 6. Plots of the first three circular harmonics of the kernel $K_{11}^{(i)}(k / q)$, Eq. (49). The corresponding simplified kernel (50) is shown for comparison (dashed line).

We define $\Gamma \equiv \Gamma^{(0,0)}$ for convenience. Its integral equation in terms of the simplified kernels is

$$
\begin{aligned}
\Gamma(p, q) & =1+\frac{\beta}{2 p} \int_{0}^{q} d k \frac{k}{q} C^{(0)}(k / p) \Gamma(k, q) \\
& +\frac{\beta}{2 p} \int_{q}^{\Lambda} d k C^{(0)}(k / p) \Gamma(k, q),
\end{aligned}
$$

which can be seen to reduce to the usual equation when $q \rightarrow 0$. The advantage of the simplified kernel $K_{11}^{(0)}$, as we now show, is that we can separate $\Gamma$ into its $q>0$ and $q<0$ parts

$$
\Gamma(k, q)=\Gamma^{<}(k, q) \theta(q-k)+\Gamma^{>}(k, q) \theta(k-q),
$$

and obtain two equations that can be solved separately and then matched. The equation for $\Gamma^{<}(p, q)$ is simply Eq. (51) when $p<q$

$$
\begin{aligned}
\Gamma^{<}(p, q) & =1+\frac{\beta}{2} \int_{0}^{q} d k \frac{k}{q}\left(\frac{\theta(p-k)}{p}+\frac{\theta(k-p)}{k}\right) \Gamma^{<}(k, q) \\
+ & \frac{\beta}{2} \int_{q}^{\Lambda} d k \frac{1}{k} \Gamma^{>}(k, q),
\end{aligned}
$$

while for $p>q$ we have

$$
\begin{aligned}
\Gamma^{>}(p, q) & =1+\frac{\beta}{2} \int_{0}^{q} d k \frac{k}{p q} \Gamma^{<}(k, q) \\
+ & \frac{\beta}{2} \int_{q}^{\Lambda} d k\left(\frac{\theta(p-k)}{p}+\frac{\theta(k-p)}{k}\right) \Gamma^{>}(k, q) .
\end{aligned}
$$

If we define

$$
\begin{aligned}
\alpha_{1}(q) & =\int_{0}^{q} d k \frac{k}{q^{2}} \Gamma^{<}(k, q), \\
\alpha_{2}(q) & =\int_{q}^{\Lambda} d k \frac{1}{k} \Gamma^{>}(k, q),
\end{aligned}
$$

we can write the two equations as

$$
\begin{aligned}
& \Gamma^{<}(p, q)=1+\frac{\beta}{2} \alpha_{2}(q) \\
& +\frac{\beta}{2} \int_{0}^{q} d k \frac{k}{q}\left(\frac{\theta(p-k)}{p}+\frac{\theta(k-p)}{k}\right) \Gamma^{<}(k, q), \\
& \Gamma^{>}(p, q)=1+\frac{\beta}{2} \frac{q}{p} \alpha_{1}(q) \\
& +\frac{\beta}{2} \int_{q}^{\Lambda} d k\left(\frac{\theta(p-k)}{p}+\frac{\theta(k-p)}{k}\right) \Gamma^{>}(k, q) .
\end{aligned}
$$

Taking two derivatives with respect to $p$ we obtain

$$
\begin{aligned}
& p^{2} \frac{\partial^{2} \Gamma^{<}}{\partial p^{2}}+2 p \frac{\partial \Gamma^{<}}{\partial p}+\frac{\beta}{2} \frac{p}{q} \Gamma^{<}=0, \\
& p^{2} \frac{\partial^{2} \Gamma^{>}}{\partial p^{2}}+2 p \frac{\partial \Gamma^{>}}{\partial p}+\frac{\beta}{2} \Gamma^{>}=0 .
\end{aligned}
$$

Thus we have obtained two independent equations for $\Gamma^{<}$and $\Gamma^{>}$. These equations are only coupled through the boundary conditions, which can be obtained from the first derivatives of equations (57) and (58). For $\Gamma^{<}$these are

$$
\begin{array}{r}
\left.\left(p \frac{\partial \Gamma^{<}}{\partial p}+\Gamma^{<}-1-\frac{\beta}{2} \alpha_{2}\right)\right|_{q}=0 \\
\left.p^{2} \frac{\partial \Gamma^{<}}{\partial p}\right|_{0}=0
\end{array}
$$


while for $\Gamma^{>}$they are

$$
\begin{array}{r}
\left.\left(p \frac{\partial \Gamma^{>}}{\partial p}+\Gamma^{>}-1\right)\right|_{\Lambda}=0, \\
p \frac{\partial \Gamma^{>}}{\partial p}+\left.\frac{\beta}{2} \alpha_{1}\right|_{q}=0 .
\end{array}
$$

The differential equations have straightforward solutions. We note the equation for $\Gamma^{>}$is again the same as the one obtained in the gap equation, and the one obtained in Ref. 18, with $q$ playing the role of the infrared cutoff. The equation for $\Gamma^{<}$is a Bessel-type equation. Their solutions are

$$
\begin{aligned}
& \Gamma^{>}=A_{+} \tilde{p}^{\eta_{+}}+A_{-} \tilde{p}^{\eta_{-}}, \\
& \Gamma^{<}=\left(\frac{p}{q}\right)^{-1 / 2}\left(c_{1} J_{1}(\sqrt{2 \beta p / q})+c_{2} Y_{1}(\sqrt{2 \beta p / q})\right) .
\end{aligned}
$$

Applying the boundary conditions we get the solutions

$$
\begin{gathered}
\Gamma^{>}=\frac{\left(\tilde{q}^{\eta_{-}}-\alpha_{1}\left(1+\eta_{-}\right)^{2}\right)\left(1+\eta_{+}\right) \tilde{p}^{\eta_{+}}}{\tilde{q}^{\eta_{-}}\left(1+\eta_{+}\right)^{2}-\tilde{q}^{\eta_{+}}\left(1+\eta_{-}\right)^{2}}+\left(\eta_{+} \leftrightarrow \eta_{-}\right), \\
\Gamma^{<}=\frac{\left(1+\beta \alpha_{2} / 2\right) J_{1}(\sqrt{2 \beta p / q})}{J_{1}(\sqrt{2 \beta})-\sqrt{\beta / 2} J_{2}(\sqrt{2 \beta})}\left(\frac{p}{q}\right)^{-1 / 2}
\end{gathered}
$$

These solutions still depend on $\alpha_{1,2}$. Plugging them into Eqs. (55) and (56) we obtain a linear system of equations for $\alpha_{1,2}$, whose solutions are

$$
\begin{aligned}
& \alpha_{1}=\frac{(2 / \beta-1)\left(\left(1+\eta_{+}\right)^{2}-\tilde{q}^{\gamma}\left(1+\eta_{-}\right)^{2}\right)+\gamma \tilde{q}^{\eta_{+}}}{\left(\left(1+\eta_{+}\right)^{2}-\tilde{q}^{\gamma}\left(1+\eta_{-}\right)^{2}\right) / \phi(\beta)+\beta^{2} / 4\left(\tilde{q}^{\gamma}-1\right)}, \\
& \alpha_{2}=\phi(\beta) \alpha_{1}-\frac{2}{\beta},
\end{aligned}
$$

with

$$
\phi(\beta)=\sqrt{2 / \beta} \frac{J_{1}(\sqrt{2 \beta})}{J_{2}(\sqrt{2 \beta})}-1 .
$$

The response function can be finally obtained as

$$
\Pi(q)=\frac{1}{\pi} \int_{0}^{q} d p \frac{p}{q} \Gamma^{<}(p, q)+\frac{1}{\pi} \int_{q}^{\Lambda} d p \Gamma^{>}(p, q) .
$$

Evaluating the integral, plugging the values of $\alpha_{1,2}$ and in the limit $q<<\Lambda$, we finally obtain

$$
\Pi(q)=\frac{\Lambda}{\pi} \frac{1}{\left(1+\eta_{+}\right)^{2}}\left[1+\left(\gamma^{2} / \phi(\beta)-1\right) \tilde{q}^{\gamma}\right] .
$$

This result reproduces the power law behavior of $\Pi(\omega=$ $0, q$ ) found numerically in Ref. 18, and shows analytically that the exponent is in fact given by $\eta_{0}=\gamma=\sqrt{1-\beta / \beta_{c}}$ with $\beta_{c}=1 / 2$. This is the excitonic transition again, in limit $\mu \rightarrow 0$ : when $\beta=\beta_{c}$ the response function becomes singular. This analytical expression provides a simple, compact expression for observables that couple to the static response function in the ladder approximation.

\section{Dynamic response}

The kernels in the general frequency dependent response are too complicated for an analytic solution. Therefore, we now solve Eq. (47) numerically by discretizing the momentum $k$ on a logarithmic mesh and solving the corresponding matrix equation by Gaussian elimination. The integration of Eq. (48) is straightforward. The results of this procedure are shown in Fig. 7. The self-energy is represented as the difference $\Delta \Pi=\Pi\left(q_{0}, q_{0}+\delta q\right)-\Pi\left(q_{0}, q_{0}\right)$ with $\delta q=q_{0}-q$ for convenience.
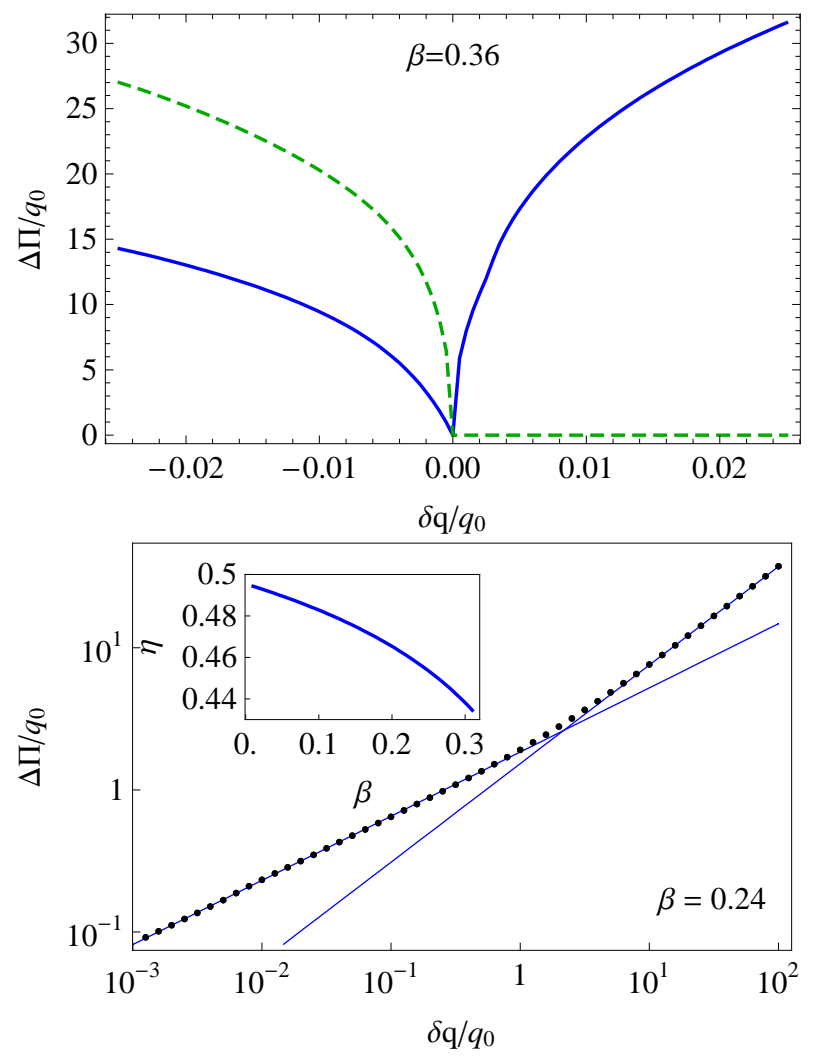

FIG. 7. Response function $\Delta \Pi\left(q_{0}, q_{0}+\delta q\right)$. a) $\Delta \Pi$ for $|\delta q|<<$ $q_{0}$ and $\beta=0.36$, real part (full line) and imaginary part (dashed line). Inset: the Kekulé phonon displacements. b) Logarithmic plot of $\Delta \Pi$ for $\beta=0.24$ and $\delta q>0$ (dotted line). The full lines are linear fits with $\eta=0.45$ for $\delta q<<q_{0}$ and $\eta_{0}=0.69$ for $\delta q>>q_{0}$.

The main result of the inclusion of finite frequency is that the response is strongly modified at $q$ close to $q_{0}$, but remains essentially the static result once $q>>q_{0}$. Fig. $7($ a) displays the real and imaginary parts of $\Delta \Pi$ for $|\delta q|<<q_{0}$. We observe a cusp at $\delta q=0$ in the real part, and a finite imaginary part for $\delta q<0$. Log plots of both sides of the real part and and the imaginary part reveal power laws as $\delta q \rightarrow 0$. A Kramers-Kronig analysis for $|\delta q|<<q_{0}$ shows that this is only consistent if $\Delta \Pi \propto(\delta q)^{\eta}$, i.e. the exponents are all the same $\mathrm{Bi}^{\underline{3}}$. Fig 7(b) shows a $\log$ plot for $\delta q>0$ where power law behavior 
is evident for $\delta q<<q_{0}$. We also observe that $\Delta \Pi$ crosses over to a different power law for $\delta q>>q_{0}$, which we identify as the static result $q^{\eta_{0} \underline{18}}$, obtained analytically in the previous section. The inset of Fig[7(b) shows that $\eta$ is $\beta$-dependent, and that it tends to the non-interacting result in Eq. (80) as $\beta \rightarrow 0$. In summary, the main features of the response function are a cusp $\left(q-q_{0}\right)^{\eta}$ around $q_{0}$, and a crossover to the static power $q^{\eta_{0}}$ for $q>>q_{0}$.

\section{EXPERIMENTAL SIGNATURES OF POWER LAW BEHAVIOR}

In the previous section we found that the mass susceptibilities have characteristic power law behavior with $\beta$ dependent exponents. We may now ask what are the experimental consequences of this. In general, these correlations can be observed in a linear response-type experiment, with a suitable probe that couples to electrons in the form of a mass. This is however difficult, as the usual experiments rather couple to the electron charge or current. To find a probe that couples to the masses we need to refer to their microscopic origins: A Kekulé distortion for $M_{1}, M_{2}$, or a sublattice antisymmetric potential for $M_{3}$.

A first proposal to measure the $\Pi_{33}$ correlator was put forward in Ref. 18 which involved placing a Coulomb impurity asymmetrically with respect to the sublattices, and measuring the sublattice charge difference with a STM tip. This measurement is difficult to perform, as it requires one to resolve the lattice structure in detail. A different possibility that we now discuss is to probe particular phonons that couple to electrons with a mass vertex. The self-energy of this type of phonons is precisely given by the mass susceptibility, which then becomes observable through the dispersion and lifetime of the phonon. These can be measured with current experimental techniques discussed below.

The phonon spectrum of the honeycomb lattice consists of six phonon branches, four in-plane and two outof-plane. Each of these phonons may couple to electrons near either Dirac point if it has momentum close to zero (a $\Gamma$ point or zone center phonon), which scatters electrons within each valley, or if it has momentum close to $K$ or $K^{\prime}$ points (a zone boundary phonon), in which case it produces intervalley scattering. The strength of the electron-phonon coupling (EPC), however, depends on how the particular displacement pattern of that phonon modifies the hopping integrals between atoms. Two modes have displacements that produce a significant EPC, and both of them are in-plane phonons. The first of these is the phonon branch of highest energy at the $\Gamma$ point, the $E_{2}$ phonon. The second is the $A_{1}$ branch at the $K$ and $K^{\prime}$ points (also the highest branch). This is a lattice distortion with a supercell of six atoms, whose displacement pattern is obtained by taking linear combinations of the displacements at $K$ and $K^{\prime}$, and is shown in the inset of Fig. 8. These two combinations couple to electrons exactly in the same way as the two components of the Kekule distortion, i.e. they couple with the mass matrices $M_{1}$ and $M_{2}$

$$
H_{e-p h, K}=F_{K} \int d^{2} r \psi^{\dagger}\left(M_{1} u_{K 1}+M_{2} u_{K 2}\right) \psi,
$$

with $F_{K}=3 \partial t / \partial a$. For this reason this phonon is also known as the Kekulé phonon ${ }^{32}$. This phonon is therefore suitable to measure the $\Pi_{11}=\Pi_{22}$ correlators.

It has also been noted that in the presence of broken $z \rightarrow-z$ symmetry, induced for example by a substrate or a perpendicular electric field, there is another mechanism that produces an EPC with out-of-plane phonons 33,34 . This is simply that atoms displaced to different positions see a different potential, and is not related to changes in hopping integrals. In particular, for the $Z O$ (out-ofplane, optical) phonon at the $\Gamma$ point, the sublattices $A$ and $\mathrm{B}$ move in opposite directions, and there is a linear coupling of the form

$$
H_{e-p h, Z O}=F_{Z O} \int d^{2} r \psi^{\dagger} M_{3} u_{Z O} \psi,
$$

with $F_{Z O} \propto E_{z}$. Therefore, in the presence of an electric field, the $Z O$ phonon can be used to measure $\Pi_{33}$. The Hamiltonian of either phonon may be expressed as

$$
H=\sum_{i} \int \frac{d^{2} q}{(2 \pi)^{2}} \omega_{i} b_{i, q}^{\dagger} b_{i, q},
$$

with creation and destruction operators defined by

$$
u_{i}=\sqrt{\frac{A_{c}}{4 \omega_{i} M}} \int \frac{d^{2} q}{(2 \pi)^{2}}\left(b_{i, q} e^{i \vec{q} \vec{r}}+b_{i, q}^{\dagger} e^{-i \vec{q} \vec{r}}\right),
$$

where $i=K 1, K 2, Z O, \omega_{K} \approx 0.17 \mathrm{eV}, \omega_{Z O} \approx 0.1 \mathrm{eV}, A_{c}$ is the unit cell area, and the dispersion of the phonons is neglected to a first approximation. A dimensionless EPC can be defined as $\lambda_{K}=F_{i}^{2} A_{c} /\left(2 M \omega_{i} v_{F}^{2}\right)$. In the case of the $A_{1}$ phonon it is estimated to be in the range $\lambda_{K} \approx 0.03-0.135,36$. We will now discuss the only the $A_{1}$ phonon, as the $Z O$ case in an electric field has the same behavior.

The phonon propagator can be obtained in terms of the self-energy as

$$
G_{p h}(\omega, q)=\frac{2 \omega_{K}}{\omega^{2}-\omega_{K}^{2}-2 \omega_{K} \Sigma(\omega, q)} .
$$

and it follows directly from the form of the electronphonon vertex Eq. (74) that the phonon self-energy is directly related to the mass susceptibility Eq. (21) via $\Sigma=\frac{\lambda_{K}}{2} \Pi$. The dispersion relation for the phonon can be obtained by solving for the pole in Eq. (78) for small $\lambda_{K}$, so that the dispersion relation is corrected to

$$
\omega(q) \approx \omega_{K}+\frac{\lambda_{K}}{2} \Pi\left(\omega_{K}, q\right) .
$$


For $\beta=0$ this is 37

$$
\omega(q)=\omega_{K}+\frac{\lambda_{K}}{4}\left(v_{F}^{2} q^{2}-\omega_{K}^{2}\right)^{1 / 2},
$$

which has a square root singularity at $q_{K}$ for $q>q_{K}$. For $q<q_{K}$ the self-energy is purely imaginary, and a finite lifetime is obtained. The Kohn anomaly is conventionally associated with a linear cusp in the dispersion, which is obtained only asymptotically for $q>>q_{K}$; the full dynamical self-energy should be used in general. Note that $q_{K}$ is approximately $2 \%$ of the $\Gamma-K$ distance in the Brillouin zone. The necessity of employing the dynamical self-energy has been emphasized before ${ }^{38-40}$, in particular in the doped case where the static approximation produces poor agreement with experiments $\stackrel{41}{1}$. Note also that a different Dirac fermion induced Kohn anomaly has been recently observed in the surface of topological insulator $\mathrm{Bi}_{2} \mathrm{Se}_{3} \stackrel{42}{ }$.

In the presence of electron-electron interactions, $\Sigma$ is modified as described in the previous section and in particular acquires power law behavior. In Fig. 8 we plot the phonon dispersion relation obtained from it for different values of $\beta$. This is given in terms of the self-energy evaluated at the phonon frequency $\omega_{K}$. To ease the comparison at different values of $\beta$, we also represent the difference

$$
\Delta \omega(q)=\omega(q)-\omega\left(q_{K}\right)=\frac{\lambda_{K}}{2}\left(\Pi\left(\omega_{K}, q\right)-\Pi\left(\omega_{K}, q_{K}\right)\right),
$$

where we have recovered physical units with $\hbar v_{F}=6.5$ $\mathrm{eV} \AA$. The values of the parameters used are $\lambda_{K}=0.1$ and $\Lambda=1.7 \mathrm{eV}$. The dispersion follows the static power law $q^{\eta_{0}(\beta)}$ for $q>>q_{K}$, and the cusp turns into $q^{\eta(\beta)}$ as discussed above. The modification of the Kohn anomaly due to interactions is rather dramatic and should be observable.

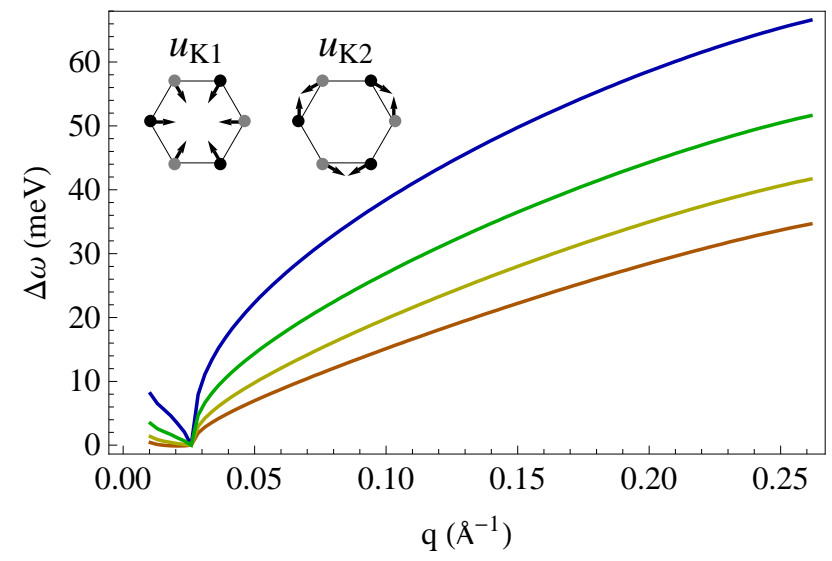

FIG. 8. $A_{1}$ phonon dispersion relation $\Delta \omega(q)$ measured from the $\mathrm{K}$ point for $\beta=0,0.1,0.2,0.3$, with higher curves corresponding to higher values of $\beta$. Note that $\omega\left(q_{K}\right)$, which depends on $\beta$, has been subtracted from each curve for an easier comparison. Inset: the Kekulé phonon displacements.
From the experimental point of view, there are several techniques available for the measurement of the $A_{1}$ phonon dispersion, and each one has its own potential difficulties. One method is to employ Electron Energy Loss Spectroscopy (EELS), which has already been used to map K-point phonon dispersions of graphene on different substrates where it behaves as quasi-freestanding ${ }^{43.44}$, such as Pt. (The absence of hybridization with the substrate is important as it would strongly change the electron band structure and the Kohn anomaly $\stackrel{45}{\underset{4}{4}}$. The problem with metallic substrates is that screening will turn the long range Coulomb interaction between graphene electrons into a short-ranged one, or may even suppress it completely. Thus a power law anomaly is not expected, which is consistent with the linear one observed in Ref. 44. An insulating substrate would be required to observe this effect.

A more indirect experiment (with insulating substrate) is to track the dependence of the 2D Raman peak with incoming laser energy. This method has been used $\frac{46}{6}$ to measure the dispersion of the $A_{1}$ phonon, but both the amount of data it yields and the range of momenta it covers is limited and not very close to the $K$ point. Finally, $\mathrm{X}$-rays are a usual tool to measure phonon dispersions in $3 \mathrm{D}$ crystals, and while it is probably challenging to obtain enough intensity from a single sheet of graphene, experiments in graphite 47,48 might be used to deduce the phonon dispersion. This approach is not straightforward because the electronic structure of graphite is different from graphene, and this must be taken into account. Nevertheless, it is encouraging to observe that precision measurements show an $A_{1}$ phonon dispersion that is not linear $\stackrel{48}{ }$.

\section{CONCLUSIONS AND DISCUSSION}

Understanding the role of interactions in graphene is a challenging problem whose solution is far from complete. One of the main purposes of this work is to bring greater attention to a new potential way of observing interaction effects: the measurement of the mass susceptibility, which, as we have shown, displays power law behavior with $\beta$-dependent exponents. Apart for unveiling a novel signature of interactions, the identification of these power laws could represent an alternative measurement of graphene's fine structure constant $\beta$.

Moreover, the observation of the static power law would also help in understanding the problem of the excitonic transition in graphene. With the experimental evidence gathered so far, there seems to be no indication of the presence of an interaction induced gap, even

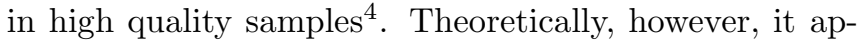
pears that suspended graphene should be, if not in the gapped phase, at least close to the transition. Since, as we have shown, the static exponent of the mass-mass response goes to zero at the critical $\beta_{c}$, a measurement of the exponent would indicate how close we are to the 
achievement of the long-sought gap.

Note also that other potential measurements of these power laws could come from spin related experiments, which we have not discussed. The inclusion of spin in the picture is straightforward: in this case, the symmetry of the Hamiltonian enlarged to SU(4), and the excitonic masses generalize to singlet and triplet versions. Because of the same symmetry argument relating CDW and Kekulé masses, any of these correlators would have the same power law behavior, so the observation of these particular spin susceptibilities may represent an alternative route to the experiments we have discussed in this work.

A final comment concerns the robustness of our result to more refined approximations schemes than the ladder summation. While other sets of diagrams may modify our quantitative predictions, it is very unlikely that the non-analytic behavior can be removed in this way. One may consider, for example, the inclusion of self-energy terms for the electron propagator ${ }^{5}$, which may produce a slow logarithmic dependence of the exponent. This could be taken into account in a similar way as was done for the gap equation 13 . The effect of static RPA screening of the Coulomb interaction will in general reduce the effective value of $\beta$ but will not change the power law itself. Finally we also note that the $1 / \mathrm{N}$ approximation does gives power law behavior for the Kekulé mass correlator ${ }^{20,21}$ (and thus the self-energy) as well.

In summary, we believe that the observation of power law correlations originating in the mass susceptibility is potentially feasible and would be an important contribution to our understanding of the problem of interactions in graphene.

\section{ACKNOWLEDGMENTS}

This work was supported by the NSF through Grant No. DMR1005035, and by US-Israel Binational Science Foundation (BSF) through Grant No. 2008256.
1 A. H. Castro Neto, F. Guinea, N. M. R. Peres, K. S. Novoselov, and A. K. Geim, Rev. Mod. Phys. 81, 109 (2009)

2 V. N. Kotov, B. Uchoa, V. M. Pereira, A. H. Castro Neto, and F. Guinea, Rev. Mod. Phys., submitted

3 J. P. Reed, B. Uchoa, Y. I. Joe, Y. Gan, D. Casa, E. Fradkin, and P. Abbamonte, Science 330, 805 (2010)

4 D. C. Elias, R. V. Gorbachev, A. S. Mayorov, S. V. Morozov, A. A. Zhukov, P. Blake, L. A. Ponomarenko, I. V. Grigorieva, K. S. Novoselov, F. Guinea, and A. K. Geim, Nat. Phys.(2011)

5 J. González, F. Guinea, and M. A. H. Vozmediano, Nucl. Phys. B 424, 595 (1994)

6 D. E. Sheehy and J. Schmalian, Phys. Rev. Lett. 99, 226803 (2007)

7 J. González, F. Guinea, and M. A. H. Vozmediano, Phys. Rev. B 59, R2474 (1999)

8 D. V. Khveshchenko, Phys. Rev. Lett. 87, 246802 (2001)

9 E. Gorbar, V. Gusynin, V. Miransky, and I. Shovkovy, Physics Letters A 313, 472 (2003)

10 D. V. Khveshchenko and W. F. Shively, Phys. Rev. B 73, 115104 (2006)

11 D. V. Khveshchenko, J. Phys: Condensed Matter 21, 075303 (2009)

12 O. V. Gamayun, E. V. Gorbar, and V. P. Gusynin, Phys. Rev. B 81, 075429 (2010)

13 J. Sabio, F. Sols, and F. Guinea, Phys. Rev. B 82, 121413 (2010)

14 C.-X. Zhang, G.-Z. Liu, and M.-Q. Huang, Phys. Rev. B 83, 115438 (2011)

15 J. E. Drut and T. A. Lähde, Phys. Rev. Lett. 102, 026802 (Jan 2009)

16 W. Armour, S. Hands, and C. Strouthos, Phys. Rev. B 81, 125105 (2010)

17 O. V. Gamayun, E. V. Gorbar, and V. P. Gusynin, Phys. Rev. B 80, 165429 (2009)

18 J. Wang, H. A. Fertig, and G. Murthy, Phys. Rev. Lett. 104, 186401 (2010)
19 J. Wang, H. A. Fertig, G. Murthy, and L. Brey, Phys. Rev. B 83, 035404 (2011)

20 J. González, Phys. Rev. B 82, 155404 (2010)

21 A. Giuliani, V. Mastropietro, and M. Porta, Phys. Rev. B $\mathbf{8 2}, 121418(2010)$

22 Note that this symmetry is only valid at low energies, as contact interactions, which are irrelevant in the RG sense, may in general break it

23 C. Chamon, Phys. Rev. B 62, 2806 (2000)

24 R. D. Pisarski, Phys. Rev. D 29, 2423 (1984)

25 T. Appelquist, D. Nash, and L. C. R. Wijewardhana, Phys. Rev. Lett. 60, 2575 (1988)

26 L. D. Landau and E. M. Lifshitz, Quantum Mechanics (Pergamon, Oxford, 1981) Chap. 5

27 V. Gusynin, A. Hams, and M. Reenders, Phys. Rev. D 63, 045025 (2001)

28 M. Franz, T. Pereg-Barnea, D. E. Sheehy, and Z. Tešanović, Phys. Rev. B 68, 024508 (2003)

29 V. P. Gusynin, D. V. Khveshchenko, and M. Reenders, Phys. Rev. B 67, 115201 (2003)

30 M. Hermele, T. Senthil, and M. P. A. Fisher, Phys. Rev. B 72, 104404 (2005)

31 Similar behavior is observed for Kohn anomalies in some one dimensional systems, see A. Luther and I. Peschel, Phys. Rev. B 9, 2911 (1974).

32 H. Suzuura and T. Ando, J. Phys. Soc. Jpn. 77, 044703 (2008)

33 J.-N. Fuchs and P. Lederer, Phys. Rev. Lett. 98, 016803 (2007)

${ }^{34}$ T. L. Linnik, cond-mat/1111.3924 29 (2011)

35 D. M. Basko and I. L. Aleiner, Phys. Rev. B 77, 041409 (2008)

36 D. M. Basko, S. Piscanec, and A. C. Ferrari, Phys. Rev. B 80, 165413 (2009)

37 S. Piscanec, M. Lazzeri, F. Mauri, A. C. Ferrari, and J. Robertson, Phys. Rev. Lett. 93, 185503 (2004)

38 M. Lazzeri and F. Mauri, Phys. Rev. Lett. 97, 266407 (2006) 
39 A. H. Castro Neto and F. Guinea, Phys. Rev. B 75, 045404 (2007)

40 W.-K. Tse, B. Y.-K. Hu, and S. Das Sarma, Phys. Rev. Lett. 101, 066401 (2008)

41 S. Pisana, M. Lazzeri, C. Casiraghi, K. S. Novoselov, A. K. Geim, A. C. Ferrari, and F. Mauri, Nat. Mater. 6, 198 (2007)

42 X. Zhu, L. Santos, R. Sankar, S. Chikara, C. . Howard, F. C. Chou, C. Chamon, and M. El-Batanouny, Phys. Rev. Lett. 107, 186102 (2011)

43 H. Yanagisawa, T. Tanaka, Y. Ishida, M. Matsue, E. Rokuta, S. Otani, and C. Oshima,
Surface and Interface Analysis 37, 133 (2005)

44 A. Politano, A. R. Marino, V. Formoso, and G. Chiarello, Carbon 50, 734 (2012)

45 A. Allard and L. Wirtz, Nano Letters 10, 4335 (2010)

46 D. L. Mafra, G. Samsonidze, L. M. Malard, D. C. Elias, J. C. Brant, F. Plentz, E. S. Alves, and M. A. Pimenta, Phys. Rev. B 76, 233407 (2007)

47 J. Maultzsch, S. Reich, C. Thomsen, H. Requardt, and P. Ordejón, Phys. Rev. Lett. 92, 075501 (2004)

48 A. Grüneis, J. Serrano, A. Bosak, M. Lazzeri, S. L. Molodtsov, L. Wirtz, C. Attaccalite, M. Krisch, A. Rubio, F. Mauri, and T. Pichler, Phys. Rev. B 80, 085423 (2009) 